\title{
SCHATTEN CLASSES AND TRACES ON COMPACT GROUPS
}

\author{
JULIO DELGADO AND MICHAEL RUZHANSKY
}

\begin{abstract}
In this paper we present symbolic criteria for invariant operators on compact topological groups $G$ characterising the Schatten-von Neumann classes $S_{r}\left(L^{2}(G)\right)$ for all $0<r \leq \infty$. Since it is known that for pseudo-differential operators criteria in terms of kernels may be less effective (Carleman's example), our criteria are given in terms of the operators' symbols defined on the noncommutative analogue of the phase space $G \times \widehat{G}$, where $G$ is a compact topological (or Lie) group and $\widehat{G}$ is its unitary dual. We also show results concerning general non-invariant operators as well as Schatten properties on Sobolev spaces. A trace formula is derived for operators in the Schatten class $S_{1}\left(L^{2}(G)\right)$. Examples are given for Bessel potentials associated to sub-Laplacians (sums of squares) on compact Lie groups, as well as for powers of the sub-Laplacian and for other non-elliptic operators on $\mathrm{SU}(2) \simeq \mathbb{S}^{3}$ and on $\mathrm{SO}(3)$.
\end{abstract}

\section{INTRODUCTION}

Let $G$ be a compact topological (or Lie) group. In this paper we address the problem of characterising the Schatten-von Neumann classes $S_{r}$ of operators on the Hilbert space $L^{2}(G)$ for $0<r \leq \infty$. To do this, we make use of the global quantization recently developed in [RT10a] and [RT13] as a noncommutative analogue of the KohnNirenberg quantization of operators on $\mathbb{R}^{n}$. For brevity in the sequel we will refer to Schatten-von Neumann classes simply as to Schatten classes.

In view of Carleman's example [Car16] recalled below, it is well understood that there is an obstruction when looking for good criteria in terms of kernels for ensuring that an operator belongs to the Schatten class $S_{r}$ below the index $r<2$.

In this paper we show that using the notion of a matrix symbol of an operator on a compact group, we can characterise or give sufficient conditions for operators in Schatten classes $S_{r}\left(L^{2}(G)\right)$ for all $0<r \leq \infty$. Moreover, our characterisations do not assume any regularity condition on the symbols. Criteria for operators with symbols in Hörmander classes have been analysed, see e.g. Shubin [Shu01, Section 27], but the regularity of symbols is required for the analysis there. Recently, there was a surge of interest in finding criteria for Schatten classes in terms of symbols with lower regularity, see e.g. [Tof06, Tof08, BT10]. In this paper we can drop the regularity assumptions completely (at least in the considered settings) due to the technique

Date: October 15, 2018.

2010 Mathematics Subject Classification. Primary 35S05; Secondary 43A75, 22E30, 47B06.

Key words and phrases. Compact Lie groups, topological groups, pseudo-differential operators, eigenvalues, trace formula, Schatten classes.

The first author was supported by Marie Curie IIF 301599 and by the Leverhulme Grant RPG2014-02. The second author was supported by EPSRC Leadership Fellowship EP/G007233/1 and by EPSRC Grant EP/K039407/1. No new data was collected or generated during the course of the research. 
of matrix quantization that we are using, instead of the traditional Kohn-Nirenberg quantization in the manifold setting.

In particular, for operators acting on $L^{2}(G)$, in Corollary 3.3 we give the characterisation of operators in the Schatten class $S_{r}\left(L^{2}(G)\right), 0<r \leq \infty$, in terms of symbols of their powers, and in Theorem 3.5 we elaborate this to provide the characterisation of left-invariant operators in terms of their symbols. In particular, the class $S_{\infty}$ of invariant bounded operators on $L^{2}(G)$ can be characterised by the uniform boundedness of the operator matrix-norms of their symbol, see (3.1). Here we note that symbolic criteria for the $L^{p}$-boundedness of operators on compact Lie groups, the Mikhlin-Hörmander multiplier theorem and its extension to non-invariant operators for $1<p<\infty$, are presented in [RW13] and in [RW15]. The nuclearity of pseudo-differential operators on the circle $\mathbb{T}^{1}$ has been recently analysed in DW13 but the situation in the present paper is much more subtle because of the necessarily appearing non-trivial multiplicities of the eigenvalues of the Laplacian on the noncommutative compact Lie groups; for $0<r \leq 1$, the $r$-nuclearity on $L^{p}$-spaces on compact Lie groups is addressed in [DR14d].

In order to illustrate the relevance of our symbolic criteria we briefly recall a classical result of Carleman (Car16]) from 1916, who has constructed a periodic continuous function $\varkappa(x)=\sum_{k=-\infty}^{\infty} c_{k} e^{2 \pi i k x}$, i.e. a continuous function on the commutative Lie group $\mathbb{T}^{1}$, for which the Fourier coefficients $c_{k}$ satisfy

$$
\sum_{k=-\infty}^{\infty}\left|c_{k}\right|^{r}=\infty \quad \text { for any } r<2
$$

Now, considering the normal operator

$$
T f=f * \varkappa
$$

acting on $L^{2}\left(\mathbb{T}^{1}\right)$ one obtains that the sequence $\left(c_{k}\right)_{k}$ forms a complete system of eigenvalues of this operator corresponding to the complete orthonormal system $\phi_{k}(x)=$ $e^{2 \pi i k x}, T \phi_{k}=c_{k} \phi_{k}$. The system $\phi_{k}$ is also complete for $T^{*}, T^{*} \phi_{k}=\overline{c_{k}} \phi_{k}$, the singular values of $T$ are given by $s_{k}(T)=\left|c_{k}\right|$ and hence

$$
\sum_{k=-\infty}^{\infty} s_{k}(T)^{r}=\infty
$$

for $r<2$. Therefore, the invariant operator $T$ is not in $S_{r}\left(L^{2}\left(\mathbb{T}^{1}\right)\right)$ for $r<2$. However, the continuous integral kernel $K(x, y)=\varkappa(x-y)$ satisfies any kind of integral condition of the form

$$
\iint|K(x, y)|^{s} d x d y<\infty
$$

due to the boundedness of $K$. This shows that already in the problem of finding sufficient conditions for an operator to belong to the Schatten class $S_{r}\left(L^{2}\left(\mathbb{T}^{1}\right)\right)$ with $r<2$, it is impossible to formulate a sufficient condition of this type for the kernel. On the other hand, it is possible to find such criteria assuming additional regularity of the kernel, the problem that was addressed in [DR14e], and a comparison between kernel and symbol criteria in those cases was done in [DR14c]. However, here we are 
interested in exploring criteria with no regularity assumptions on the kernel (and on the symbol).

In this work we will be looking to establish conditions imposed on symbols instead of kernels characterising the operators from Schatten classes. We give examples of our results on the torus $\mathbb{T}^{n}$, on $\mathrm{SU}(2)$ and on $\mathrm{SO}(3)$. Since $\mathrm{SU}(2) \simeq \mathbb{S}^{3}$ are globally diffeomorphic and isomorphic, with the product of matrices in $\mathrm{SU}(2)$ corresponding to the quaternionic product on $\mathbb{S}^{3}$, our results immediately extend to $\mathbb{S}^{3}$ since the symbolic calculus is stable under global diffeomorphisms preserving the groups structure (see [RT10a, Section 12.5] or [RT13]). Moreover, in view of the recently resolved Poincaré conjecture, any simply-connected closed 3-manifold $M$ is globally diffeomorphic to $\mathbb{S}^{3}$ inducing the corresponding group structure on $M$. Thus, our criteria extend to such $M$ as well with no changes in formulations.

We observe that most of the results of this paper hold true for compact topological groups, without assuming the differential Lie group structure, which we need only in the statements involving differential operators. We follow [RT10a, Chapter 7] in assuming that the unit element set $\{e\}$ is closed so that the topological groups are Hausdorff, and also refer to it for other background details on compact topological groups and their representation theory.

Moreover, we can observe that the notion of matrix-valued symbols on compact groups and our criteria become instrumental and can be used as a tool in questions which may be formulated intrinsically on the group without referring to a particular quantization.

For example, in Theorem 3.11, we conclude (by a simple argument) that Schatten classes for left-invariant operators on Sobolev spaces $H^{s}(G)$ are independent with respect to the order $s$ of the space.

To give another specific example of conclusions independent of the quantization used, let $\mathcal{L}$ and $\mathcal{L}_{\text {sub }}$ denote the (negative-definite) Laplacian and the sub-Laplacian on the group $G=\mathrm{SU}(2)$, respectively, (or, with the same conclusion, on the group $\mathrm{SO}(3)$, or on the quaternionic sphere $\left.\mathbb{S}^{3}\right)$. Then we will show in Section 4 that for $0<r<\infty$, we have

$$
(I-\mathcal{L})^{-\alpha / 2} \in S_{r}\left(L^{2}\left(\mathbb{S}^{3}\right)\right) \quad \text { if and only if } \quad \alpha r>3,
$$

while

$$
\left(I-\mathcal{L}_{\text {sub }}\right)^{-\alpha / 2} \in S_{r}\left(L^{2}\left(\mathbb{S}^{3}\right)\right) \quad \text { if and only if } \quad \alpha r>4 .
$$

Here the powers $\left(I-\mathcal{L}_{\text {sub }}\right)^{-\alpha / 2}$ are well-defined since $\mathcal{L}_{\text {sub }}$ is hypoelliptic, which follows from Hörmander's sum of the squares theorem (see also Greenfield and Wallach GW73] for a general framework for this, and [RTW14] for the associated hypoelliptic symbolic calculus on compact Lie groups). We also give an example for the family of operators

$$
\mathcal{H}_{\gamma}=i D_{3}-\gamma\left(D_{1}^{2}+D_{2}^{2}\right), 0<\gamma<\infty,
$$

which are not covered by Hörmander's sum of the squares theorem. The criterion established in Theorem 3.5 allows one to easily conclude that the operators $(I+$ $\left.\mathcal{H}_{\gamma}\right)^{-\alpha / 2}$ are never in Schatten classes for $0<\gamma \leq 1$, while for $\gamma>1$ show that it is in the class $S_{r}$ if and only if $\alpha r>4$.

In Section 2 we make a short introduction to the global quantization on compact groups. In Section 3 we give characterisations for general operators in Schatten classes 
$S_{r}\left(L^{2}(G)\right)$ for $0<r<\infty$, elaborate this in the case of left-invariant operators, and deduce several corollaries, including a criterion for Bessel potentials. In Proposition 3.12 we look at the example of powers of sums of squares of vector fields satisfying Hörmander's commutators condition to a certain order. In Section 4 we give examples of our results on the torus $\mathbb{T}^{n}$, and for powers of the Laplacian and sub-Laplacian on $\mathrm{SU}(2) \simeq \mathbb{S}^{3}$ and on $\mathrm{SO}(3)$. In Section 5 we derive a trace formula for left-invariant operators.

The authors would like to thank Jens Wirth for discussions and remarks, and Marius Măntoiu for a comment.

\section{Preliminaries}

In this section we recall some basic facts about the global matrix quantization on a compact topological/Lie group $G$. Let $\widehat{G}$ denote the set of equivalence classes of continuous irreducible unitary representations of $G$. Since $G$ is compact, the set $\widehat{G}$ is discrete. For $[\xi] \in \widehat{G}$, by choosing a basis in the representation space of $\xi$, we can view $\xi$ as a matrix-valued function $\xi: G \rightarrow \mathbb{C}^{d_{\xi} \times d_{\xi}}$, where $d_{\xi}$ is the dimension of the representation space of $\xi$. By the Peter-Weyl theorem the collection

$$
\left\{\sqrt{d_{\xi}} \xi_{i j}: 1 \leq i, j \leq d_{\xi},[\xi] \in \widehat{G}\right\}
$$

is the orthonormal basis of $L^{2}(G)$. If $f \in L^{1}(G)$ we define its global Fourier transform at $\xi$ by

$$
\mathcal{F}_{G} f(\xi) \equiv \widehat{f}(\xi):=\int_{G} f(x) \xi(x)^{*} d x
$$

where $d x$ is the normalised Haar measure on $G$. Thus, if $\xi$ is a matrix representation, we have $\widehat{f}(\xi) \in \mathbb{C}^{d_{\xi} \times d_{\xi}}$. The Fourier inversion formula is a consequence of the PeterWeyl theorem, so that we have

$$
f(x)=\sum_{[\xi] \in \widehat{G}} d_{\xi} \operatorname{Tr}(\xi(x) \widehat{f}(\xi)) .
$$

Given a sequence of matrices $a(\xi) \in \mathbb{C}^{d_{\xi} \times d_{\xi}}$, we can define

$$
\left(\mathcal{F}_{G}^{-1} a\right)(x):=\sum_{[\xi] \in \widehat{G}} d_{\xi} \operatorname{Tr}(\xi(x) a(\xi)) .
$$

This series can be interpreted distributionally or absolutely depending on the growth of (the Hilbert-Schmidt norms of ) $a(\xi)$. We refer to [RT10a] for further discussion of this background material. The Parseval identity takes the form

$$
\|f\|_{L^{2}(G)}=\left(\sum_{[\xi] \in \widehat{G}} d_{\xi}\|\widehat{f}(\xi)\|_{\mathrm{HS}}^{2}\right)^{1 / 2}, \quad \text { where }\|\widehat{f}(\xi)\|_{\mathrm{HS}}^{2}=\operatorname{Tr}\left(\widehat{f}(\xi) \widehat{f}(\xi)^{*}\right),
$$

which gives the norm on $\ell^{2}(\widehat{G})$.

For each $[\xi] \in \widehat{G}$, the matrix elements of $\xi$ are the eigenfunctions for the Laplacian $\mathcal{L}_{G}$ (or the Casimir element of the universal enveloping algebra), with the same 
eigenvalue which we denote by $-\lambda_{[\xi]}^{2}$, so that we have

$$
-\mathcal{L}_{G} \xi_{i j}(x)=\lambda_{[\xi]}^{2} \xi_{i j}(x) \quad \text { for all } 1 \leq i, j \leq d_{\xi}
$$

The weight for measuring the decay or growth of Fourier coefficients in this setting is

$$
\langle\xi\rangle:=\left(1+\lambda_{[\xi]}^{2}\right)^{\frac{1}{2}}
$$

the eigenvalues of the elliptic first-order pseudo-differential operator $\left(I-\mathcal{L}_{G}\right)^{\frac{1}{2}}$.

For a linear continuous operator $A$ from $C^{\infty}(G)$ to $\mathcal{D}^{\prime}(G)$ we define its matrixvalued symbol $\sigma_{A}(x, \xi) \in \mathbb{C}^{d_{\xi} \times d_{\xi}}$ by

$$
\sigma_{A}(x, \xi):=\xi(x)^{*}(A \xi)(x) \in \mathbb{C}^{d_{\xi} \times d_{\xi}},
$$

where $A \xi(x) \in \mathbb{C}^{d_{\xi} \times d_{\xi}}$ is understood as $(A \xi(x))_{i j}=\left(A \xi_{i j}\right)(x)$, i.e. by applying $A$ to each component of the matrix $\xi(x)$. Then one has ([RT10a], [RT13]) the global quantization

$$
A f(x)=\sum_{[\xi] \in \widehat{G}} d_{\xi} \operatorname{Tr}\left(\xi(x) \sigma_{A}(x, \xi) \widehat{f}(\xi)\right)
$$

in the sense of distributions, and the sum is independent of the choice of a representation $\xi$ from each equivalence class $[\xi] \in \widehat{G}$. If $A$ is a linear continuous operator from $C^{\infty}(G)$ to $C^{\infty}(G)$, the series (2.7) is absolutely convergent and can be interpreted in the pointwise sense. We will also write $A=\operatorname{Op}\left(\sigma_{A}\right)$ for the operator $A$ given by the formula (2.7). The symbol $\sigma_{A}$ can be interpreted as a matrix-valued function on $G \times \widehat{G}$. We refer to [RT10a, RT13] for the consistent development of this quantization and the corresponding symbolic calculus.

If the operator $A$ is left-invariant then its symbol $\sigma_{A}$ does not depend on $x$. In this case we will often just say that $A$ is invariant.

\section{Schatten Classes on $L^{2}(G)$}

In this section we study symbolic criteria for Schatten classes $S_{r}\left(L^{2}(G)\right)$ in terms of their full matrix-valued symbols, in particular we characterise invariant operators in the Schatten classes $S_{r}\left(L^{2}(G)\right)$.

We recall that if $A \in \Psi^{m}(G)$ is a pseudo-differential operators in Hörmander's class $\Psi^{m}(G)$, i.e. if all of its localisations to $\mathbb{R}^{n}$ are pseudo-differential operators with symbols in the class $S_{1,0}^{m}\left(\mathbb{R}^{n}\right)$, then the matrix-symbol of $A$ satisfies

$$
\left\|\sigma_{A}(x, \xi)\right\|_{o p} \leq C\langle\xi\rangle^{m} \quad \text { for all } x \in G,[\xi] \in \widehat{G} .
$$

Here and everywhere $\|\cdot\|_{o p}$ denotes the operator norm of the matrix multiplication by the matrix $\sigma_{A}(x, \xi)$. For this fact, see e.g. [RT10a, Lemma 10.9.1] or [RT13, and for the complete characterisation of Hörmander classes $\Psi^{m}(G)$ in terms of matrixvalued symbols see also [RTW14. In particular, this motivates the appearance of the operator norms of the matrix-valued symbols. However, since $\sigma_{A}$ is in general a matrix, other matrix norms become useful as well. 
We recall that if $H$ is a complex Hilbert space, a linear compact operator $A: H \rightarrow$ $H$ belongs to the Schatten class $S_{r}(H)$ if

$$
\sum_{n=1}^{\infty}\left(s_{n}(A)\right)^{r}<\infty
$$

where $s_{n}(A)$ denote the singular values of $A$, i.e. the eigenvalues of $|A|=\sqrt{A^{*} A}$ with multiplicities counted. If $1 \leq r<\infty$ the class $S_{r}(H)$ becomes a Banach space endowed with the norm

$$
\|A\|_{S_{r}}=\left(\sum_{n=1}^{\infty}\left(s_{n}(A)\right)^{r}\right)^{\frac{1}{r}}
$$

If $0<r<1$ the identity above only defines a quasi-norm with respect to which $S_{r}(H)$ is complete. The class $S_{2}(H)$ and $S_{1}(H)$ are usually known as the class of Hilbert-Schmidt operators and the trace class, respectively.

In the case of $r=\infty$ we can put $\|A\|_{S_{\infty}}$ to be the operator norm of the bounded operator $A: L^{2}(G) \rightarrow L^{2}(G)$. In this case it can be easily seen by the Plancherel formula that for an invariant operator $A$, we have

$$
A \in S_{\infty}\left(L^{2}(G)\right) \quad \text { if and only if } \sup _{[\xi] \in \widehat{G}}\left\|\sigma_{A}(\xi)\right\|_{o p}<\infty,
$$

see e.g. [RT10a, Section 10.5]. So, in the sequel we can assume that $0<r<\infty$. The following lemma is a consequence of the definition of Schatten classes:

Lemma 3.1. Let $A: H \rightarrow H$ be a linear compact operator. Let $0<r, t<\infty$. Then $A \in S_{r}$ if and only if $|A|^{\frac{r}{t}} \in S_{t}$. Moreover, $\|A\|_{S_{r}}^{r}=\left\||A|^{\frac{r}{t}}\right\|_{S_{t}}^{t}$.

We will denote by $\|\sigma(x, \xi)\|_{S_{r}}$ the Schatten-norm of order $r$ of the matrix $\sigma(x, \xi) \in$ $\mathbb{C}^{d_{\xi} \times d_{\xi}}$, for $x, \xi$ fixed, viewed as a linear mapping on $\mathbb{C}^{d_{\xi}}$.

We start by giving a simple criterion for Hilbert-Schmidt operators. On general $L^{2}(\mu)$ spaces the Hilbert-Schmidt operators are characterised by the square integrability of the kernel with respect to the product measure $\mu \otimes \mu$. It is also well known that one can translate this characterisation in terms of symbols for pseudo-differential operators on the Euclidean space. We state below a characterisation of Hilbert-Schmidt operators in terms of the matrix-valued symbol on compact Lie groups. This underlines the natural role played by the matrix-valued symbol on compact Lie groups. The norm of $L^{2}(G \times \widehat{G})$ can be defined by

$$
\|\sigma\|_{L^{2}(G \times \widehat{G})}=\left(\int_{G} \sum_{[\xi] \in \widehat{G}} d_{\xi}\|\sigma(x, \xi)\|_{\mathrm{HS}}^{2} d x\right)^{\frac{1}{2}} .
$$

This is a natural norm in view of the Parseval identity (2.4).

Proposition 3.2. Let $G$ be a compact Lie group. Let $A: L^{2}(G) \rightarrow L^{2}(G)$ be a linear continuous operator with matrix-valued symbol $\sigma_{A}(x, \xi)$. Then $A$ is a Hilbert-Schmidt operator if and only if $\sigma_{A} \in L^{2}(G \times \widehat{G})$. Moreover, $\|A\|_{\mathrm{HS}}=\left\|\sigma_{A}\right\|_{L^{2}(G \times \widehat{G})}$. 
In particular, if $\sigma_{A}(\xi)$ depends only on $\xi$, then $A$ is Hilbert-Schmidt if and only if its symbol $\sigma_{A}$ satisfies

$$
\sum_{[\xi] \in \widehat{G}} d_{\xi}\left\|\sigma_{A}(\xi)\right\|_{\mathrm{HS}}^{2}<\infty .
$$

Proof. The kernel of $A$ is given by

$$
K(x, y)=\sum_{[\xi] \in \widehat{G}} d_{\xi} \operatorname{Tr}\left(\xi(x) \sigma_{A}(x, \xi) \xi(y)^{*}\right) .
$$

We have $\|A\|_{\mathrm{HS}}^{2}=\int_{G} \int_{G}|K(x, y)|^{2} d x d y=\int_{G} \int_{G}\left|K\left(x, x z^{-1}\right)\right|^{2} d x d z$. From (3.3) we obtain

$$
\begin{aligned}
K\left(x, x z^{-1}\right) & =\sum_{[\xi] \in \widehat{G}} d_{\xi} \operatorname{Tr}\left(\xi(x) \sigma_{A}(x, \xi) \xi\left(x z^{-1}\right)^{*}\right) \\
& =\sum_{[\xi] \in \widehat{G}} d_{\xi} \operatorname{Tr}\left(\xi\left(x z^{-1}\right)^{*} \xi(x) \sigma_{A}(x, \xi)\right) \\
& =\sum_{[\xi] \in \widehat{G}} d_{\xi} \operatorname{Tr}\left(\xi(z) \sigma_{A}(x, \xi)\right) \\
& =\mathcal{F}_{G}^{-1} \sigma_{A}(x, \cdot)(z),
\end{aligned}
$$

with $\mathcal{F}_{G}^{-1}$ defined in (2.3). We have formally

$$
\begin{aligned}
\int_{G} \int_{G}\left|K\left(x, x z^{-1}\right)\right|^{2} d x d z=\int_{G} \int_{G}\left|\mathcal{F}_{G}^{-1} \sigma_{A}(x, \cdot)(z)\right|^{2} d z d x & \\
& =\int_{G} \sum_{[\xi] \in \widehat{G}} d_{\xi}\left\|\sigma_{A}(x, \xi)\right\|_{\mathrm{HS}}^{2} d x=\left\|\sigma_{A}\right\|_{L^{2}(G \times \widehat{G})}^{2} .
\end{aligned}
$$

The second equality is obtained from the Parseval identity (2.4).

As a consequence of Lemma 3.1 with $t=2$, and Proposition 3.2 we have:

Corollary 3.3. Let $G$ be a compact Lie group. Let $A: L^{2}(G) \rightarrow L^{2}(G)$ be a linear continuous operator with matrix-valued symbol $\sigma_{A}(x, \xi)$. Let $0<r<\infty$. Then $A \in S_{r}\left(L^{2}(G)\right)$ if and only if

$$
\sum_{[\xi] \in \widehat{G}} d_{\xi} \int_{G}\left\|\sigma_{|A|^{\frac{r}{2}}}(x, \xi)\right\|_{\mathrm{HS}}^{2} d x<\infty .
$$

In particular, for invariant operators, i.e. for operators $A$ with matrix-valued symbols $\sigma_{A}(\xi)$ depending only on $\xi$, we have that $A \in S_{r}\left(L^{2}(G)\right)$ if and only if

$$
\sum_{[\xi] \in \widehat{G}} d_{\xi}\left\|\sigma_{|A|^{\frac{r}{2}}}(\xi)\right\|_{\mathrm{HS}}^{2}<\infty .
$$

With the purpose of characterising invariant operators belonging to a Schatten class $S_{r}\left(L^{2}(G)\right)$ for $0<r<\infty$ we first establish a simple fact required for the characterisation in the case of invariant operators: 
Lemma 3.4. Let $A: L^{2}(G) \rightarrow L^{2}(G)$ be a linear continuous operator with matrixvalued symbol $\sigma_{A}(\xi)$ depending only on $\xi$. Then, for each $[\xi] \in \widehat{G}$ and $0<r<\infty$ we have

$$
\sigma_{|A|^{r}}(\xi)=\left|\sigma_{A}(\xi)\right|^{r}
$$

Proof. Since $A$ is invariant, we have

$$
\sigma_{A^{*} A}(\xi)=\sigma_{A^{*}}(\xi) \sigma_{A}(\xi),
$$

which can be expressed as $\sigma_{|A|^{2}}(\xi)=\left|\sigma_{A}(\xi)\right|^{2}$. In general, since the operator $|A|$ is formally self-adjoint, its symbol $\sigma_{|A|}(\xi)$ is self-adjoint for every $\xi$; indeed, we have $\sigma_{|A|}(\xi)=\sigma_{|A|^{*}}(\xi)=\sigma_{|A|}(\xi)^{*}$, the last equality because of the left-invariance of $|A|$. Consequently, we can diagonalise $\sigma_{|A|}(\xi)$ by a unitary transformation, which means that we can assume that $\sigma_{|A|}(\eta)$ is diagonal for some $\eta \in[\xi]$. Consequently, the positivity of the symbol (of the positive operator $|A|$ ) implies that $\sigma_{|A|^{r}}(\eta)=\left|\sigma_{A}(\eta)\right|^{r}$ for any $0<r<\infty$. Going back to the representation $\xi$ we obtain the statement.

We can now state the characterisation for invariant operators in $S_{r}\left(L^{2}(G)\right)$. We note here that since it is formulated for invariant operators, we do not need the differential structure on $G$, so that all the arguments in the proof actually work in the more general setting of compact topological groups.

Theorem 3.5. Let $G$ be a compact topological group and let $0<r<\infty$. Let $A: L^{2}(G) \rightarrow L^{2}(G)$ be a linear compact operator with matrix-valued symbol $\sigma_{A}(\xi)$ depending only on $\xi$. Then $A \in S_{r}\left(L^{2}(G)\right)$ if and only if

$$
\sum_{[\xi] \in \widehat{G}} d_{\xi}\left\|\sigma_{A}(\xi)\right\|_{S_{r}}^{r}<\infty .
$$

Proof. By Corollary $3.3 A \in S_{r}\left(L^{2}(G)\right)$ if and only if $\sum_{[\xi] \in \widehat{G}} d_{\xi}\left\|\sigma_{|A| \frac{r}{2}}(\xi)\right\|_{S_{2}}^{2}<\infty$. But Lemma 3.4 gives us $\left\|\sigma_{|A|^{\frac{r}{2}}}(\xi)\right\|_{S_{2}}=\left\|\left|\sigma_{A}(\xi)\right|^{\frac{r}{2}}\right\|_{S_{2}}$. From Lemma 3.1 applied to the matrix-symbol $\sigma_{A}(\xi)$ for each $\xi$, we obtain $\left\|\left|\sigma_{A}(\xi)\right|^{\frac{r}{2}}\right\|_{S_{2}}^{2}=\left\|\left|\sigma_{A}(\xi)\right|^{r}\right\|_{S_{1}}$. Applying Lemma 3.1 once more gives us $\left\|\left|\sigma_{A}(\xi)\right|^{r}\right\|_{S_{1}}=\left\|\sigma_{A}(\xi)\right\|_{S_{r}}^{r}$, concluding the proof.

Remark 3.6. The expression $\sum_{[\xi] \in \widehat{G}} d_{\xi}\left\|\sigma_{A}(\xi)\right\|_{S_{r}}^{r}$ appearing in the condition of Theorem 3.5 characterising invariant operators in Schatten classes comes from the norm

$$
\left\|\sigma_{A}\right\|_{\ell_{s c h}^{r}}:=\left(\sum_{[\xi] \in \widehat{G}} d_{\xi}\left\|\sigma_{A}(\xi)\right\|_{S_{r}}^{r}\right)^{1 / r} .
$$

For the analysis of spaces with norms $\left\|\sigma_{A}\right\|_{\ell_{s c h}^{r}}$ we refer to Hewitt and Ross [HR70, Section 31] or Edwards [Edw72, Section 2.14]. Because of the Hausdorff-Young inequality for the Fourier transform in these spaces (see Kunze [Kun58]) we get the following one-sided criteria for invariant operators:

Corollary 3.7. Let $G$ be a compact Lie group and let the left-invariant operator $A$ be bounded on $L^{2}(G)$. Then it is of the form $A f=f * k$ with $k \in \mathcal{D}^{\prime}(G)$ such that $\sup _{[\xi] \in \widehat{G}}\|\widehat{k}(\xi)\|_{o p}<\infty$. Moreover, we have the following properties:

(i) if $k \in L^{p}(G)$ with $1 \leq p \leq 2$, then $A \in S_{p^{\prime}}\left(L^{2}(G)\right)$ with $\frac{1}{p}+\frac{1}{p^{\prime}}=1$; 
(ii) if $A \in S_{p}\left(L^{2}(G)\right)$ with $1 \leq p \leq 2$, then $k \in L^{p^{\prime}}(G)$ with $\frac{1}{p}+\frac{1}{p^{\prime}}=1$.

Proof. The representation $A f=f * k$ follows from the Schwartz kernel theorem, while the $L^{2}$-boundedness implies that $\sup _{[\xi] \in \widehat{G}}\|\widehat{k}(\xi)\|_{o p}<\infty$ by (3.1). We also have $\sigma_{A}(\xi)=\widehat{k}(\xi)$. Consequently, both properties (i) and (ii) follow from Theorem 3.5 and from the Hausdorff-Young inequalities for the Fourier transform mapping between $L^{p}(G)$ and spaces with the norm

$$
\|\widehat{f}\|_{\ell_{s c h}^{p^{\prime}}}:=\left(\sum_{[\xi] \in \widehat{G}} d_{\xi}\|\widehat{f}(\xi)\|_{S_{p^{\prime}}}^{p^{\prime}}\right)^{1 / p^{\prime}}
$$

see also (3.4).

The following lemma has been proved in DR14a and it will be useful here to deduce other consequences.

Lemma 3.8. Let $G$ be a compact Lie group. Then we have

$$
\sum_{[\xi] \in \widehat{G}} d_{\xi}^{2}\langle\xi\rangle^{-s}<\infty \quad \text { if and only if } \quad s>\operatorname{dim} G .
$$

This Lemma 3.8 and Theorem 3.5 yield the following corollary:

Corollary 3.9. Let $G$ be a compact Lie group of dimension $n$ and let $A$ be an operator with symbol $\sigma(\xi)$. Let $0<r<\infty$. Suppose that $\|\sigma(\xi)\|_{S_{r}} \leq C d_{\xi}^{1 / r}\langle\xi\rangle^{-\frac{s}{r}}$ with some $s>n$. Then $A \in S_{r}\left(L^{2}(G)\right)$.

Before we give several examples of this corollary in Section 4, let us apply it to the Bessel potentials on $G$, also showing the sharpness of the obtained orders. As before, we denote by $\mathcal{L}_{G}$ a Laplacian on $G$, see Stein [Ste70] for a general discussion on Laplacians on compact Lie groups. We recall that $\mathcal{L}_{G}$ is a negative-definite second order bi-invariant elliptic differential operators, the eigenvalues of $\left(I-\mathcal{L}_{G}\right)^{1 / 2}$ are denoted by $\langle\xi\rangle$, and the symbol of its powers is

$$
\sigma_{\left(I-\mathcal{L}_{G}\right)^{-\alpha / 2}}(\xi)=\langle\xi\rangle^{-\alpha} I_{d_{\xi}},
$$

where $I_{d_{\xi}} \in \mathbb{C}^{d_{\xi} \times d_{\xi}}$ is the identity matrix. In this case we then readily calculate $\left\|\sigma_{\left(I-\mathcal{L}_{G}\right)^{-\alpha / 2}}(\xi)\right\|_{S_{r}}=d_{\xi}^{1 / r}\langle\xi\rangle^{-\alpha}$. In particular, this, together with the following proposition, shows that the orders in Corollary 3.9 are sharp.

Proposition 3.10. Let $G$ be a compact Lie group of dimension $n$. Then $\left(I-\mathcal{L}_{G}\right)^{-\alpha / 2}$ is in the Schatten class $S_{r}\left(L^{2}(G)\right), 0<r<\infty$, if and only if $\alpha r>n$.

Proof. We have $\left\|\sigma_{\left(I-\mathcal{L}_{G}\right)^{-\alpha / 2}}(\xi)\right\|_{S_{r}}=d_{\xi}^{1 / r}\langle\xi\rangle^{-\alpha}$, so that

$$
\sum_{[\xi] \in \widehat{G}} d_{\xi}\left\|\sigma_{\left(I-\mathcal{L}_{G}\right)^{-\alpha / 2}}(\xi)\right\|_{S_{r}}^{r}=\sum_{[\xi] \in \widehat{G}} d_{\xi}^{2}\langle\xi\rangle^{-\alpha r},
$$

and Proposition 3.10 follows from combining the criteria in Theorem 3.5 and in Lemma 3.8. 
We note that the statement of Proposition 3.10 can be extended to a more general setting on compact manifolds, see [DR14b], as well as [DR14e] for a comparison, so the more interesting setting for us here is that of non-elliptic operators that we address in the next section.

We will now give some consequences regarding Schatten classes on Sobolev spaces. The notion of global symbol and the characterisation given by Theorem 3.5 of Schatten classes for left-invariant operators provide a simple proof of the independence with respect to the scale of Sobolev spaces $H^{s}(G)$ (defined by their localisations being in $\left.H^{s}\left(\mathbb{R}^{n}\right)\right)$.

Theorem 3.11. Let $G$ be a compact Lie group, $0<r<\infty$ and $s \in \mathbb{R}$. Let $A$ : $H^{s}(G) \rightarrow H^{s}(G)$ be a linear bounded operator with the matrix-valued symbol $\sigma_{A}(\xi)$ depending only on $\xi$. Then

$$
A \in S_{r}\left(H^{s}(G)\right) \text { if and only if } \sum_{[\xi] \in \widehat{G}} d_{\xi}\left\|\sigma_{A}(\xi)\right\|_{S_{r}}^{r}<\infty .
$$

Consequently, if $s_{1}, s_{2} \in \mathbb{R}$ and $A$ is a left-invariant operator bounded on $A: H^{s_{1}}(G) \rightarrow$ $H^{s_{1}}(G)$ and $A: H^{s_{2}}(G) \rightarrow H^{s_{2}}(G)$, then

$$
A \in S_{r}\left(H^{s_{1}}(G)\right) \text { if and only if } A \in S_{r}\left(H^{s_{2}}(G)\right) \text {. }
$$

Proof. We observe that if $A u=\lambda u$ with $u \in H^{s}(G)$, writing

$$
u=(I-\mathcal{L})^{-\frac{s}{2}} f
$$

with $f \in L^{2}(G)$ and $\mathcal{L}$ denoting a Laplacian on $G$, we get

$$
A(I-\mathcal{L})^{-\frac{s}{2}} f=\lambda(I-\mathcal{L})^{-\frac{s}{2}} f
$$

Hence we have

$$
\tilde{A} f=\lambda f, \text { with } \tilde{A}=(I-\mathcal{L})^{\frac{s}{2}} A(I-\mathcal{L})^{-\frac{s}{2}} .
$$

Thus $\tilde{A}: L^{2}(G) \rightarrow L^{2}(G)$ is bounded on $L^{2}(G)$ and

$$
\sigma_{\tilde{A}}(\xi)=\sigma_{(I-\mathcal{L})^{\frac{s}{2}}}(\xi) \sigma_{A}(\xi) \sigma_{(I-\mathcal{L})^{-\frac{s}{2}}}(\xi)=\sigma_{A}(\xi)
$$

because all these operators are left-invariant and $\sigma_{(I-\mathcal{L})^{\frac{s}{2}}}(\xi)=\langle\xi\rangle^{s} I_{\mathrm{d}_{\xi}}$ is diagonal. Therefore, using Theorem 3.5, we obtain

$$
\begin{aligned}
A \in S_{r}\left(H^{s}(G)\right) & \Longleftrightarrow \tilde{A} \in S_{r}\left(L^{2}(G)\right) \\
& \Longleftrightarrow \sum_{[\xi] \in \widehat{G}} d_{\xi}\left\|\sigma_{\tilde{A}}(\xi)\right\|_{S_{r}}^{r}<\infty \\
& \Longleftrightarrow \sum_{[\xi] \in \widehat{G}} d_{\xi}\left\|\sigma_{A}(\xi)\right\|_{S_{r}}^{r}<\infty,
\end{aligned}
$$

completing the proof.

Similar to Proposition 3.10 we can give a condition for powers of sub-Laplacians. Let $G$ be a compact Lie group of dimension $n$ and let

$$
\mathcal{L}_{\text {sub }}=X_{1}^{2}+\cdots+X_{k}^{2}
$$

be the sum of squares of left-invariant vector fields $X_{1}, \ldots, X_{k}$, for which we assume that the Hörmander commutator condition is satisfied of order $\varkappa \in \mathbb{N}$, i.e. the 
commutators of length $\varkappa$ span the Lie algebra of $G$. Since the operator $\mathcal{L}_{\text {sub }}$ is formally self-adjoint, we can choose the bases in the representation spaces in such a way that its symbol is diagonal, and we denote

$$
\sigma_{I-\mathcal{L}_{\text {sub }}}(\xi)=\operatorname{diag}\left\{\nu_{1}^{2}(\xi), \ldots, \nu_{d_{\xi}}^{2}(\xi)\right\}
$$

for some $\nu_{j}(\xi) \geq 0$. Then, following a-priori estimates by Rothschild and Stein [RS76], it was shown in [GR15, Proposition 3.1] that there exist constants $c, C>0$ such that

$$
c\langle\xi\rangle^{1 / \varkappa} \leq \nu_{j}(\xi) \leq C\langle\xi\rangle
$$

holds for all $[\xi] \in \widehat{G}$ and all $1 \leq j \leq d_{\xi}$.

Proposition 3.12. Let $G$ be a compact Lie group of dimension n. Let

$$
\mathcal{L}_{\text {sub }}=X_{1}^{2}+\cdots+X_{k}^{2}
$$

be the sum of squares of left-invariant vector fields $X_{1}, \ldots, X_{k}$ satisfying the Hörmander commutator condition of order $\varkappa$. Then $\left(I-\mathcal{L}_{\text {sub }}\right)^{-\alpha / 2}$ is in the Schatten class $S_{r}\left(L^{2}(G)\right), 0<r<\infty$, provided that $\alpha r>\varkappa n$.

Proof. The statement follows readily from Theorem 3.5 using (3.5). Namely, we can write

$$
\sum_{[\xi] \in \widehat{G}} d_{\xi}\left\|\sigma_{\left(I-\mathcal{L}_{\text {sub }}\right)^{-\frac{\alpha}{2}}}(\xi)\right\|_{S_{r}}^{r}=\sum_{[\xi] \in \widehat{G}} \sum_{j=1}^{d_{\xi}} d_{\xi} \nu_{j}(\xi)^{-\alpha r} \leq C \sum_{[\xi] \in \widehat{G}} d_{\xi}^{2}\langle\xi\rangle^{-\frac{\alpha r}{\varkappa}}
$$

Therefore, (3.6) is finite for $\alpha r>\varkappa n$ in view of Lemma 3.8,

For the case of the Laplacian, we have $\varkappa=1$, so that we recover the condition in Proposition 3.10. For $\varkappa \geq 2$, however, the condition $\alpha r>\varkappa n$ in Proposition [3.12 can be improved in a number of cases. This requires a more careful analysis of the maximal weight lattice and will be addressed elsewhere. In Corollary 4.6, following explicit calculations, we give its improvement in the case of the group $\mathrm{SU}(2)$.

Assuming $\varkappa=2$ for simplicity, we note that the corresponding condition $\alpha r>2 n$ in Proposition 3.12 is related to subelliptic estimates for the sub-Laplacian that we now briefly indicate. Indeed, since $\mathcal{L}_{\text {sub }}$ is a sum of squares associated to a system of vector fields satisfying the Hörmander condition of order $\varkappa=2$, we have

$$
\|u\|_{H^{1}} \leq C\left\|\left(I-\mathcal{L}_{s u b}\right) u\right\|_{L^{2}}
$$

see e.g. Rothschild and Stein [RS76]. It follows then that $\left\|\left(I-\mathcal{L}_{\text {sub }}\right)^{-1} u\right\|_{H^{1}} \leq$ $C\|u\|_{L^{2}}$, and hence, by interpolation,

$$
\left\|\left(I-\mathcal{L}_{\text {sub }}\right)^{-s} u\right\|_{H^{s}} \leq C\|u\|_{L^{2}}
$$

for any $s \geq 0$. Before we apply this, we observe that on the other hand, if $\delta$ is the delta-distribution at the unit element of the group, we have

$$
\mathcal{F}_{G}\left(\left(I-\mathcal{L}_{\text {sub }}\right)^{-\frac{\beta}{2}} \delta\right)(\xi)=\sigma_{\left(I-\mathcal{L}_{\text {sub }}\right)^{-\frac{\beta}{2}}}(\xi) \widehat{\delta}(\xi)=\operatorname{diag}\left\{\nu_{j}(\xi)^{-\beta}\right\} I_{d_{\xi}}
$$


Taking $\beta=\frac{\alpha r}{2}$, we get

$$
\begin{aligned}
\left\|\mathcal{F}_{G}\left(\left(I-\mathcal{L}_{\text {sub }}\right)^{-\frac{\alpha r}{4}} \delta\right)(\xi)\right\|_{\ell^{2}(\widehat{G})}^{2} & =\sum_{[\xi] \in \widehat{G}} d_{\xi}\left\|\mathcal{F}_{G}\left(\left(I-\mathcal{L}_{\text {sub }}\right)^{-\frac{\alpha r}{4}} \delta\right)(\xi)\right\|_{\mathrm{HS}}^{2} \\
& =\sum_{[\xi] \in \widehat{G}} \sum_{j=1}^{d_{\xi}} d_{\xi} \nu_{j}(\xi)^{-\alpha r} .
\end{aligned}
$$

By Plancherel Theorem, and combining this with the equality in (3.6), we get

$$
\sum_{[\xi] \in \widehat{G}} d_{\xi}\left\|\sigma_{\left(I-\mathcal{L}_{\text {sub }}\right)^{-\frac{\alpha}{2}}}(\xi)\right\|_{S_{r}}^{r}=\left\|\left(I-\mathcal{L}_{\text {sub }}\right)^{-\frac{\alpha r}{4}} \delta\right\|_{L^{2}(G)}^{2} .
$$

Since the Laplacian and the sub-Laplacian commute, using (3.7), we get

$$
\left\|\left(I-\mathcal{L}_{\text {sub }}\right)^{-\frac{\alpha r}{4}} \delta\right\|_{L^{2}} \leq C\|\delta\|_{H^{-\frac{\alpha r}{4}}}<\infty
$$

for $\frac{\alpha r}{4}>\frac{n}{2}$, i.e. for $\alpha r>2 n$, the same order as in Proposition 3.12, Although the subelliptic estimate (3.7) is sharp, the possibility of improving the orders for Schatten classes, such as the one that we obtain in Corollary 4.6, can be explained by the fact that we only need to apply (3.7) to the delta-distribution, in which case the Sobolev order can be actually better.

\section{ExAmples ON $\mathbb{T}^{n}, \mathrm{SU}(2) \simeq \mathbb{S}^{3}$ AND $\mathrm{SO}(3)$}

We now give some examples of our results on the torus $\mathbb{T}^{n}, \mathrm{SU}(2)$ and $\mathrm{SO}(3)$. In particular, this shows that the notion of the matrix-valued symbol becomes instrumental and can be used as a tool for deriving properties of operators defined intrinsically on the group.

4.1. The torus $\mathbb{T}^{n}$. We start with a few simple observations in the case of the torus. If $G=\mathbb{T}^{n}=\mathbb{R}^{n} / \mathbb{Z}^{n}$, we have $\widehat{\mathbb{T}^{n}} \simeq \mathbb{Z}^{n}$, and the collection $\left\{\xi_{k}(x)=e^{2 \pi i x \cdot k}\right\}_{k \in \mathbb{Z}^{n}}$ is the orthonormal basis of $L^{2}\left(\mathbb{T}^{n}\right)$, and all $d_{\xi_{k}}=1$. If an operator $A$ is invariant on $\mathbb{T}^{n}$, its symbol becomes $\sigma_{A}\left(\xi_{k}\right)=\xi_{k}(x)^{*} A \xi_{k}(x)=A \xi_{k}(0)$. In general, on the torus we will often simplify the notation by identifying $\widehat{\mathbb{T}^{n}}$ with $\mathbb{Z}^{n}$, and thus writing $\xi \in \mathbb{Z}^{n}$ instead of $\xi_{k} \in \mathbb{Z}^{n}$. The toroidal quantization

$$
A f(x)=\sum_{\xi \in \mathbb{Z}^{n}} e^{2 \pi i x \cdot \xi} \sigma_{A}(x, \xi) \widehat{f}(\xi)
$$

has been analysed extensively in [RT10b] and it is a special case of (2.7), where we have identified, as noted, $\widehat{\mathbb{T}^{n}}$ with $\mathbb{Z}^{n}$. As a consequence of Theorem 3.5 and Corollary 3.9 on the torus, we obtain:

Corollary 4.1. Let $A: L^{2}\left(\mathbb{T}^{n}\right) \rightarrow L^{2}\left(\mathbb{T}^{n}\right)$ be a linear continuous operator with symbol $\sigma_{A}(\xi)$ depending only on $\xi$. Let $0<r<\infty$, then $A$ belongs to $S_{r}\left(L^{2}\left(\mathbb{T}^{n}\right)\right)$ if and only if its symbol $\sigma_{A}$ satisfies

$$
\sum_{\xi \in \mathbb{Z}^{n}}\left|\sigma_{A}(\xi)\right|^{r}<\infty .
$$


In particular, $A$ belongs to $S_{r}\left(L^{2}\left(\mathbb{T}^{n}\right)\right)$ provided that

$$
\left|\sigma_{A}(\xi)\right| \leq C\langle\xi\rangle^{-\frac{s}{r}}
$$

for some $s>n$.

Remark 4.2. Corollary 4.1 implies that a necessary condition for nuclearity $(r=1)$ on $L^{2}\left(\mathbb{T}^{n}\right)$ for operators with symbol only depending on $\xi$ is the continuity of the corresponding kernel. Indeed, since

$$
K(x, y)=\sum_{\xi \in \mathbb{Z}^{n}} e^{i(x-y) \xi} \sigma_{A}(\xi),
$$

the continuity of $K$ follows from the fact that $\sigma_{A} \in \ell^{1}\left(\mathbb{Z}^{n}\right)$. An analogue of this property on general $G$ was given in Corollary 3.7 .

The result concerning the Bessel potentials in Proposition 3.10 in the case of $S_{r}\left(L^{2}\left(\mathbb{T}^{n}\right)\right)$ becomes as follows:

Proposition 4.3. Let $\Delta$ be the Laplacian on the torus $\mathbb{T}^{n}$ and let $0<r<\infty$. Then $(I-\Delta)^{-\frac{\alpha}{2}}$ belongs to $S_{r}\left(L^{2}\left(\mathbb{T}^{n}\right)\right)$ if and only if $\alpha r>n$.

Proof. We give a direct simple proof of this. The symbol of the operator $T=(I-$ $\Delta)^{-\frac{\alpha}{2}}$ is positive, hence $T$ being a multiplier operator, it is positive definite and $|T|=\sqrt{T^{*} T}=T$. Thus, the singular values of $T$ agree with the values of its symbol $\langle\xi\rangle^{-\alpha}$. Therefore, $T \in S_{r}\left(L^{2}\left(\mathbb{T}^{n}\right)\right.$ if and only if $\alpha r>n$.

4.2. The groups $\mathrm{SU}(2) \simeq \mathbb{S}^{3}$ and $\mathrm{SO}(3)$. We now consider the case of the noncommutative group $G=\mathrm{SU}(2)$, the group of the unitary $2 \times 2$ matrices of determinant one. The same results as given below hold for the 3 -sphere $\mathbb{S}^{3}$ if we use the identification $\mathrm{SU}(2) \simeq \mathbb{S}^{3}$, with the matrix multiplication in $\mathrm{SU}(2)$ corresponding to the quaternionic product on $\mathbb{S}^{3}$, with the corresponding identification of the symbolic calculus, see [RT10a, Section 12.5]. Consequently, the results below extend to any simply-connected closed 3-manifold in view of the resolved Poincaré conjecture, see the discussion in the introduction. More generally, they can be extended to general closed manifolds, see DR14b.

The details of the global quantization (2.7) on $\mathrm{SU}(2)$ have been worked out in [RT10a, Chapter 12], to which we also refer for the details on the representation theory of the group $G=\mathrm{SU}(2)$. In this case, we can enumerate the elements of its dual as $\widehat{G} \simeq \frac{1}{2} \mathbb{N}_{0}$, with $\mathbb{N}_{0}=\{0\} \cup \mathbb{N}$, so that

$$
\widehat{\mathrm{SU}(2)}=\left\{\left[t^{\ell}\right]: t^{\ell} \in \mathbb{C}^{(2 \ell+1) \times(2 \ell+1)}, \ell \in \frac{1}{2} \mathbb{N}_{0}\right\} .
$$

The dimension of each $t^{\ell}$ is $d_{t^{\ell}}=2 \ell+1$, and there are explicit formulae for $t^{\ell}$ as functions of Euler angles in terms of the so-called Legendre-Jacobi polynomials, see [RT10a, Chapter 11]. The Laplacian on $\mathrm{SU}(2)$ has eigenvalues $\lambda_{t^{\ell}}^{2}=\ell(\ell+1)$, so that we have $\left\langle t^{\ell}\right\rangle \approx \ell$. If $A: L^{2}(\mathrm{SU}(2)) \rightarrow L^{2}(\mathrm{SU}(2))$ is a continuous linear operator, its matrix-symbol is denfined by

$$
\sigma_{A}(x, \ell) \equiv \sigma_{A}\left(x, t^{\ell}\right):=t^{\ell}(x)^{*} A t^{\ell}(x), \quad \ell \in \frac{1}{2} \mathbb{N}_{0} .
$$

Corollary 3.9 in this case becomes: 
Corollary 4.4. Let $A: L^{2}(\mathrm{SU}(2)) \rightarrow L^{2}(\mathrm{SU}(2))$ be an invariant operator with matrix symbol $\sigma_{A}(\ell)$. Let $s>3$ and let $0<r<\infty$. If there is a constant $C>0$ such that

$$
\left\|\sigma_{A}(\ell)\right\|_{S_{r}} \leq C \ell^{\frac{1-s}{r}}
$$

for all $\ell \in \frac{1}{2} \mathbb{N}$, then $A \in S_{r}\left(L^{2}(\mathrm{SU}(2))\right)$.

We now discuss examples of two operators with diagonal symbols, the Laplacian and the sub-Laplacian.

If $\mathcal{L}_{\mathrm{SU}(2)}$ denotes the Laplacian on $\mathrm{SU}(2)$, we have $\mathcal{L}_{\mathrm{SU}(2)} t_{m n}^{\ell}(x)=-\ell(\ell+1) t_{m n}^{\ell}(x)$ for all $\ell, m, n$ and $x \in G$, so that the symbol of $I-\mathcal{L}_{\mathrm{SU}(2)}$ is given by

$$
\sigma_{I-\mathcal{L}_{\mathrm{SU}(2)}}(x, \ell)=(1+\ell(\ell+1)) I_{2 \ell+1},
$$

where $I_{2 \ell+1} \in \mathbb{C}^{(2 \ell+1) \times(2 \ell+1)}$ is the identity matrix. Hence, $\sigma_{I-\mathcal{L}_{\mathrm{SU}(2)}}(x, \ell)$ is diagonal

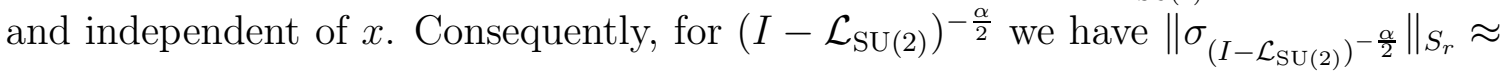
$\ell^{-\alpha} \ell^{\frac{1}{r}}$. Therefore, by Corollary 4.4. $\left(I-\mathcal{L}_{\mathrm{SU}(2)}\right)^{-\frac{\alpha}{2}}$ is in $S_{r}\left(L^{2}(\mathrm{SU}(2))\right)$ provided that $\ell^{-\alpha} \leq C \ell^{-\frac{s}{r}}$ for $s>3$, which agrees with Proposition 3.10.

Corollary 4.5. Let $0<r<\infty$. Then the operator $\left(I-\mathcal{L}_{\mathrm{SU}(2)}\right)^{-\frac{\alpha}{2}}$ is in $S_{r}\left(L^{2}(\mathrm{SU}(2))\right)$ if and only if $\alpha>\frac{3}{r}$.

To give a slightly different example, we shall now consider the group $\mathrm{SO}(3)$ of the $3 \times 3$ real orthogonal matrices of determinant one. For the details of the representation theory and the global quantization of $\mathrm{SO}(3)$ we refer the reader to RT10a, Chapter $12]$. The dual in this case can be identified as $\widehat{G} \simeq \mathbb{N}_{0}$, so that

$$
\widehat{\mathrm{SO}(3)}=\left\{\left[t^{\ell}\right]: t^{\ell} \in \mathbb{C}^{(2 \ell+1) \times(2 \ell+1)}, \ell \in \mathbb{N}_{0}\right\} .
$$

The dimension of each $t^{\ell}$ is $d_{t^{\ell}}=2 \ell+1$. The Laplacian on $\mathrm{SO}(3)$ has eigenvalues $\lambda_{t^{\ell}}^{2}=\ell(\ell+1)$, so that we have $\left\langle t^{\ell}\right\rangle \approx \ell$. By the same argument as above, Corollary 4.5 also holds for the Laplacian on $\mathrm{SO}(3)$.

Let us fix three invariant vector fields $D_{1}, D_{2}, D_{3}$ on $\mathrm{SO}(3)$ corresponding to the derivatives with respect to the Euler angles. We refer to [RT10a, Chapter 11] for the explicit formulae for these. However, for our purposes here we note that the subLaplacian $\mathcal{L}_{\text {sub }}=D_{1}^{2}+D_{2}^{2}$, with an appropriate choice of basis in the representation spaces, has the diagonal symbol given by

$$
\sigma_{\mathcal{L}_{\text {sub }}}(\ell)_{m n}=\left(m^{2}-\ell(\ell+1)\right) \delta_{m n}, \quad m, n \in \mathbb{Z},-\ell \leq m, n \leq \ell,
$$

where $\delta_{m n}$ is the Kronecker delta, and where it is customary to let $m, n$ run from $-\ell$ to $\ell$ rather than from 0 to $2 \ell+1$. The operator $\mathcal{L}_{\text {sub }}$ is a second order hypoelliptic operator and we can define the powers $\left(I-\mathcal{L}_{\text {sub }}\right)^{-\alpha / 2}$. These are pseudo-differential operators with symbols

$$
\sigma_{\left(I-\mathcal{L}_{s u b}\right)^{-\alpha / 2}}(\ell)_{m n}=\left(1+\ell(\ell+1)-m^{2}\right)^{-\alpha / 2} \delta_{m n},
$$

with $m, n \in \mathbb{Z},-\ell \leq m, n \leq \ell$. We now have

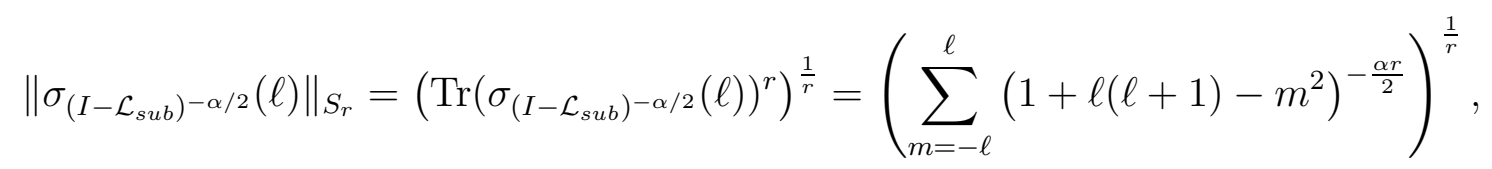


where $\ell \in \mathbb{N}_{0}$. Comparing with the integral

$$
\int_{-R}^{R}\left(1+R^{2}-x^{2}\right)^{-\frac{\alpha r}{2}} d x \approx C R^{-\frac{\alpha r}{2}} \int_{0}^{R}(1+R-x)^{-\frac{\alpha r}{2}} d x \approx C R^{-\frac{\alpha r}{2}}
$$

for $\alpha r>2$ and large $R$, it follows that $\sum_{m=-\ell}^{\ell}\left(1+\ell(\ell+1)-m^{2}\right)^{-\frac{\alpha r}{2}}$ is of order $\ell^{-\frac{\alpha r}{2}}$. Hence, $\left\|\sigma_{\left(I-\mathcal{L}_{\text {sub }}\right)^{-\alpha / 2}}(\ell)\right\|_{S_{r}}$ is of order $\ell^{-\frac{\alpha}{2}}$. Therefore, we have

$$
\sum_{[\xi] \in \widehat{\operatorname{SO}(3)}} d_{\xi}\left\|\sigma_{\left(I-\mathcal{L}_{\text {sub }}\right)^{-\alpha / 2}}(\xi)\right\|_{S_{r}}^{r} \approx C \sum_{\ell \in \mathbb{N}} \ell^{1-\frac{\alpha r}{2}}
$$

and as a consequence of Theorem 3.5, we obtain

Corollary 4.6. Let $0<r<\infty$. Then the operator $\left(I-\mathcal{L}_{\text {sub }}\right)^{-\frac{\alpha}{2}}$ belongs to the Schatten class $S_{r}\left(L^{2}(\mathrm{SO}(3))\right)$ if and only if $\alpha>\frac{4}{r}$. The same conclusion holds if we replace $\mathrm{SO}(3)$ by $\mathrm{SU}(2)$ or by $\mathbb{S}^{3}$ (with a quaternionic sub-Laplacian).

We also present another example of an operator (on $\mathrm{SO}(3)$ ) which is not covered by Hörmander's sum of squares theorem. Namely, we consider the following family of 'Schrödinger operators'

$$
\mathcal{H}_{\gamma}=i D_{3}-\gamma\left(D_{1}^{2}+D_{2}^{2}\right)
$$

for a parameter $0<\gamma<\infty$. For $\gamma=1$ it was shown in [RTW14] that $\mathcal{H}_{1}+c I$ is globally hypoelliptic if and only if $0 \notin\{c+\ell(\ell+1)-m(m+1): \ell \in \mathbb{N}, m \in \mathbb{Z},|m| \leq \ell\}$.

The matrix-symbol of $I+\mathcal{H}_{\gamma}$ is given by

$$
\sigma_{I+\mathcal{H}_{\gamma}}(\ell)_{m n}=\left(1+m-\gamma m^{2}+\gamma \ell(\ell+1)\right) \delta_{m n}, \quad m, n \in \mathbb{Z},-\ell \leq m, n \leq \ell,
$$

where as before $\delta_{m n}$ is the Kronecker delta, and we let $m, n$ run from $-\ell$ to $\ell$ rather than from 0 to $2 \ell+1$. Similarly to the case of $\gamma=1$ above, for $\gamma \geq 1$ one shows that the second order differential operator $I+\mathcal{H}_{\gamma}$ is globally hypoelliptic and its powers are pseudo-differential operators with symbols

$$
\sigma_{\left(I+\mathcal{H}_{\gamma}\right)^{-\alpha / 2}}(\ell)_{m n}=\left(1+m-\gamma m^{2}+\gamma \ell(\ell+1)\right)^{-\alpha / 2} \delta_{m n}
$$

with $m, n \in \mathbb{Z},-\ell \leq m, n \leq \ell$. We now have

$$
\left\|\sigma_{\left(I+\mathcal{H}_{\gamma}\right)^{-\alpha / 2}}(\ell)\right\|_{S_{r}}^{r}=\operatorname{Tr}\left|\sigma_{\left(I+\mathcal{H}_{\gamma}\right)^{-\alpha / 2}}(\ell)\right|^{r}=\sum_{m=-\ell}^{\ell}\left|1+m-\gamma m^{2}+\gamma \ell(\ell+1)\right|^{-\frac{\alpha r}{2}}
$$

where $\ell \in \mathbb{N}_{0}$. In order to estimate this sum, we consider the integral

$$
\int_{-R}^{R}\left|1+x-\gamma x^{2}+\gamma R^{2}+\gamma R\right|^{-\frac{\alpha r}{2}} d x
$$

Using the inequality $-R \leq x \leq R$ and from the identity $1+x-\gamma x^{2}+\gamma R^{2}+\gamma R=$ $1+(R+x)+\gamma\left(R^{2}-x^{2}\right)+\gamma R-R$, we get $1+x-\gamma x^{2}+\gamma R^{2}+\gamma R \geq(\gamma-1) R$. In particular, if $\gamma>1$ we obtain

$$
\int_{-R}^{R}\left(1+x-\gamma x^{2}+\gamma R^{2}+\gamma R\right)^{-\frac{\alpha r}{2}} d x \approx C R^{-\frac{\alpha r}{2}}
$$


for large $R$. Therefore, for $\gamma>1$,

$$
\sum_{m=-\ell}^{\ell}\left(1+m-\gamma m^{2}+\gamma \ell(\ell+1)\right)^{-\frac{\alpha r}{2}}
$$

is of order $\ell^{-\frac{\alpha r}{2}}$. Hence, $\left\|\sigma_{\left(I+\mathcal{H}_{\gamma}\right)^{-\alpha / 2}}(\ell)\right\|_{S_{r}}$ is of order $\ell^{-\frac{\alpha}{2}}$ in this case. Consequently, if $\gamma>1$ we obtain

$$
\sum_{[\xi] \in \widehat{\mathrm{SO}(3)}} d_{\xi}\left\|\sigma_{\left(I+\mathcal{H}_{\gamma}\right)^{-\alpha / 2}}(\xi)\right\|_{S_{r}}^{r} \approx C \sum_{\ell \in \mathbb{N}} \ell^{1-\frac{\alpha r}{2}}
$$

Thus, if $\gamma>1$ we get

$$
\left(I+\mathcal{H}_{\gamma}\right)^{-\alpha / 2} \in S_{r} \text { if and only if } \alpha r>4 \quad(\gamma>1) .
$$

Now, let us consider the case $0<\gamma \leq 1$. If $-1 \notin\left\{\gamma \ell(\ell+1)-\gamma m^{2}+m\right): \ell \in \mathbb{N}_{0}, m \in$ $\mathbb{Z},|m| \leq \ell\}$, or, more generally, if $-c \notin\left\{\gamma \ell(\ell+1)-\gamma m^{2}+m\right): \ell \in \mathbb{N}_{0}, m \in \mathbb{Z},|m| \leq$ $\ell\}$, the operator $c I+\mathcal{H}_{\gamma}$ is invertible, and we can define its real powers as above. Arguing as above, the corresponding modification of the integral (4.6) does not decay with respect to $R$, so by the characterisation of Schatten classes in Theorem 3.5 we get

$$
\left(c I+\mathcal{H}_{\gamma}\right)^{-\alpha / 2} \notin S_{r} \text { for all } 0<r<\infty \text { and } \alpha \in \mathbb{R} \quad(0<\gamma \leq 1) .
$$

A similar result holds then also on $\mathrm{SU}(2) \simeq \mathbb{S}^{3}$.

\section{A trace formula in the trace Class $S_{1}\left(L^{2}(G)\right)$}

In this section we give trace formulae for operators on compact topological groups. We start by recalling the definition of the trace of operators on Hilbert spaces.

Let $T: H \rightarrow H$ be an operator in $S_{1}(H)$ and let $\left\{\phi_{k}\right\}_{k}$ be any orthonormal basis for the Hilbert space $H$. Then, the series $\sum_{k=1}^{\infty}\left\langle T \phi_{k}, \phi_{k}\right\rangle_{H}$ is absolutely convergent and the sum is independent of the choice of the orthonormal basis $\left\{\phi_{k}\right\}_{k}$. Thus, we can define the trace $\operatorname{Tr}(T)$ of any linear operator $T: H \rightarrow H$ in $S_{1}(H)$ by

$$
\operatorname{Tr}(T)=\sum_{k=1}^{\infty}\left\langle T \phi_{k}, \phi_{k}\right\rangle_{H}
$$

where $\left\{\phi_{k}: k=1,2, \ldots\right\}$ is any orthonormal basis for $H$.

We will apply the definition above to the orthonormal basis of $L^{2}(G)$ given by

$$
\left\{\sqrt{d_{\xi}} \xi_{i j}: 1 \leq i, j \leq d_{\xi},[\xi] \in \widehat{G}\right\}
$$

Theorem 5.1. Let $G$ be a compact topological group. Let $A$ be a left-invariant operator in $S_{1}\left(L^{2}(G)\right)$ with matrix-valued symbol $\sigma_{A}(\xi)$. Then its trace is given by

$$
\operatorname{Tr} A=\sum_{[\xi] \in \widehat{G}} d_{\xi} \operatorname{Tr}\left(\sigma_{A}(\xi)\right) .
$$


Proof. Let $A$ be a left-invariant operator which belongs to $S_{1}\left(L^{2}(G)\right)$. We denote $\sigma=\sigma_{A}$, and the formula

$$
K(x, y)=\sum_{[\xi] \in \widehat{G}} d_{\xi} \operatorname{Tr}\left(\xi(x) \sigma(\xi) \xi(y)^{*}\right)
$$

represents the integral kernel of $A$. We will calculate $\left\langle A \eta_{\ell m}, \eta_{\ell m}\right\rangle_{L^{2}(G)}$ for $1 \leq \ell, m \leq$ $d_{\eta},[\eta] \in \widehat{G}$. We observe that

$$
\operatorname{Tr}\left(\xi(x) \sigma(\xi) \xi(y)^{*}\right)=\sum_{i, j=1}^{d_{\xi}}(\xi(x) \sigma(\xi))_{i j} \overline{\xi(y)}_{i j}
$$

Hence

$$
\begin{aligned}
A \eta_{\ell m}(x) & =\int_{G} \sum_{[\xi] \in \widehat{G}} d_{\xi} \operatorname{Tr}\left(\xi(x) \sigma(\xi) \xi(y)^{*}\right) \eta_{\ell m}(y) d y \\
& =\int_{G} \sum_{[\xi] \in \widehat{G}} d_{\xi} \sum_{i, j=1}^{d_{\xi}}(\xi(x) \sigma(\xi))_{i j} \overline{\xi(y)_{i j}} \eta_{\ell m}(y) d y \\
& =\int_{G} \sum_{[\xi] \in \widehat{G}} d_{\xi} \sum_{i, j=1}^{d_{\xi}} \sum_{k=1}^{d_{\xi}} \xi(x)_{i k} \sigma(\xi)_{k j} \overline{\xi(y)_{i j}} \eta_{\ell m}(y) d y
\end{aligned}
$$

Now, since $\left\langle\xi_{i k}, \eta_{\ell m}\right\rangle_{L^{2}(G)}=d_{\xi}^{-1} \delta_{(i, j),(\ell, m)}$ by the orthonormality of the system $\left\{\sqrt{d_{\xi}} \xi_{i j}\right\}$, we obtain

$$
\left\langle A \eta_{\ell m}, \eta_{\ell m}\right\rangle_{L^{2}(G)}=d_{\eta} d_{\eta}^{-1} \sigma(\eta)_{m m}=\sigma(\eta)_{m m}
$$

Therefore,

$$
\sum_{[\eta] \in \widehat{G}} \sum_{\ell, m}\left\langle A \eta_{\ell m}, \eta_{\ell m}\right\rangle_{L^{2}(G)}=\sum_{[\eta] \in \widehat{G}} \sum_{\ell, m} \sigma(\eta)_{m m}=\sum_{[\eta] \in \widehat{G}} d_{\eta} \operatorname{Tr}(\sigma(\eta)),
$$

concluding the proof.

\section{REFERENCES}

[BT10] E. Buzano and J. Toft. Schatten-von Neumann properties in the Weyl calculus. J. Funct. Anal., 259(12):3080-3114, 2010.

[Car16] T. Carleman. Über die Fourierkoeffizienten einer stetigen Funktion. Acta Math., 41(1):377-384, 1916. Aus einem Brief an Herrn A. Wiman.

[DR14a] A. Dasgupta and M. Ruzhansky. Gevrey functions and ultradistributions on compact Lie groups and homogeneous spaces. Bull. Sci. Math., 138(6):756-782, 2014.

[DR14b] J. Delgado and M. Ruzhansky. Fourier multipliers, symbols and nuclearity on compact manifolds. to appear in J. Anal. Math., arXiv:1404.6479, 2014.

[DR14c] J. Delgado and M. Ruzhansky. Kernel and symbol criteria for Schatten classes and $r$ nuclearity on compact manifolds. C. R. Acad. Sci. Paris, Ser. I 352:779-784, 2014.

[DR14d] J. Delgado and M. Ruzhansky. $L^{p}$-nuclearity, traces, and Grothendieck-Lidskii formula on compact Lie groups. J. Math. Pures. Appl., 102:153-172, 2014.

[DR14e] J. Delgado and M. Ruzhansky. Schatten classes on compact manifolds: Kernel conditions. J. Funct. Anal., 267:772-798, 2014. 
[DW13] J. Delgado and M. W. Wong. $L^{p}$-nuclear pseudodifferential operators on $\mathbb{Z}$ and $\mathbb{S}$. Proc. Amer. Math. Soc., 141(4):3935-3942, 2013.

[Edw72] R. E. Edwards. Integration and harmonic analysis on compact groups. Cambridge Univ. Press, London, 1972. London Mathematical Society Lecture Note Series, No. 8.

[GR15] C. Garetto and M. Ruzhansky. Wave equation for sums of squares on compact Lie groups. J. Differential Equations, 258(12):4324-4347, 2015.

[GW73] S. J. Greenfield and N. R. Wallach. Remarks on global hypoellipticity. Trans. Amer. Math. Soc., 183:153-164, 1973.

[HR70] E. Hewitt and K. A. Ross. Abstract harmonic analysis. Vol. II: Structure and analysis for compact groups. Analysis on locally compact Abelian groups. Die Grundlehren der mathematischen Wissenschaften, Band 152. Springer-Verlag, New York, 1970.

[Kun58] R. A. Kunze. $L_{p}$ Fourier transforms on locally compact unimodular groups. Trans. Amer. Math. Soc., 89:519-540, 1958.

[RS76] L. P. Rothschild and E. M. Stein. Hypoelliptic differential operators and nilpotent groups. Acta Math., 137(3-4):247-320, 1976.

[RT10a] M. Ruzhansky and V. Turunen. Pseudo-differential operators and symmetries. Background analysis and advanced topics, volume 2 of Pseudo-Differential Operators. Theory and Applications. Birkhäuser Verlag, Basel, 2010.

[RT10b] M. Ruzhansky and V. Turunen. Quantization of pseudo-differential operators on the torus. J. Fourier Anal. Appl., 16(6):943-982, 2010.

[RT13] M. Ruzhansky and V. Turunen. Global quantization of pseudo-differential operators on compact Lie groups, SU(2), 3-sphere, and homogeneous spaces. Int. Math. Res. Not. IMRN, (11):2439-2496, 2013.

[RTW14] M. Ruzhansky, V. Turunen, and J. Wirth. Hörmander class of pseudo-differential operators on compact Lie groups and global hypoellipticity. J. Fourier Anal. Appl., 20(3):476499, 2014.

[RW13] M. Ruzhansky and J. Wirth. On multipliers on compact Lie groups. Funct. Anal. Appl., 47(1):87-91, 2013.

[RW15] M. Ruzhansky and J. Wirth. $L^{p}$ Fourier multipliers on compact Lie groups. Math. Z., 280(3-4):621-642, 2015.

[Shu01] M. A. Shubin. Pseudodifferential operators and spectral theory. Springer-Verlag, Berlin, second edition, 2001. Translated from the 1978 Russian original by Stig I. Andersson.

[Ste70] E. M. Stein. Topics in harmonic analysis related to the Littlewood-Paley theory. Annals of Mathematics Studies, No. 63. Princeton University Press, Princeton, N.J., 1970.

[Tof06] J. Toft. Schatten-von Neumann properties in the Weyl calculus, and calculus of metrics on symplectic vector spaces. Ann. Global Anal. Geom., 30(2):169-209, 2006.

[Tof08] J. Toft. Schatten properties for pseudo-differential operators on modulation spaces. In Pseudo-differential operators, volume 1949 of Lecture Notes in Math., pages 175-202. Springer, Berlin, 2008.

Department of Mathematics, Imperial College London, 180 Queen's Gate, London SW7 2AZ, UNITED KINGDOM

E-mail address: j.delgado@imperial.ac.uk

Department of Mathematics, Imperial College London, 180 Queen's Gate, London SW7 2AZ, United Kingdom

E-mail address: m.ruzhansky@imperial.ac.uk 